\title{
Stiffness of an elliptical cracked shaft under bending
}

\author{
Wei Wu ${ }^{a}{ }^{*}$, Junjie $\mathrm{Gu}^{\mathrm{b}}$ and Gang Guo ${ }^{\mathrm{c}}$
}

School of Mechanical and Power Engineering, East China University of Science and Technology, Shanghai 200237, China

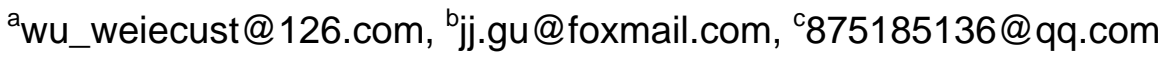

Keywords: Stiffness, Rotor, Elliptical front crack.

\begin{abstract}
Elliptical front crack has been found to be more accurate and realistic for modeling the surface crack. The stiffness of an elliptical cracked rotor submitted to bending are derived and solved based on fracture mechanics method and multi-parameter fitting technique. "Breathing effect" occurs due to gravity when rotating. It is found that stiffness values are reduced obviously, taking into account the crack depth and shape factors.
\end{abstract}

\section{Introduction}

Rotating machinery, whose main components are rotors, bearings and so on, plays a significant role in modern industry. Its safety of running causes great attention extensively. As the most common failure, rotor cracks would cause catastrophic accidents. Hence, it is necessary to study the dynamic behaviors of a rotor with cracks since it may supply theoretic support for crack detection and identification. It is well known that the presence of a crack in rotating components would decrease its stiffness. Straight front cracks have been studied in some works, but the front of cracks is elliptical in fact.

The crack model presented by Dimarogonas et al. [1-2] in 1980s has been widely accepted and adopted in dynamic analysis of cracked shafts and rotors. Tori bio et al [3] published a critical review of stress intensity factor solutions numerically for elliptical crack. Jun and Eun [4] found that the intensity factor equal to zero along the front crack line to divide the open and closed area. And Rubio [5-6] employed the intensity factor solutions to formulate flexibility of an elliptical cracked rotor under bending. Papadopoulos [7] published an excellent review paper, with detailed works from solving the stiffness of cracked rotor to dynamic vibration and crack detection.

For the accurate surface crack modeling, the elliptical front shape should be taken into account. There are four general kinds of stiffness model to describe the variation, but not precise enough. So in the present work, crack breathing behaviors and time-varying stiffness were modeled for each crack element using Crack Closure Line Position method. Fracture mechanics method combined with closed-form stress intensity factors result in accurate stiffness in each position when rotating. And that would be useful to study the behaviors.

\section{Elliptical crack modeling}

An elliptical cracked crack subjected to the forces, $Q_{x}$ and $Q_{y}$ is illustrated in Fig.1. The forces induce the additional deflections due to the crack estimated by using fracture mechanics concepts as described in Ref. [1]. In the present study, expressions for the stiffness of a crack with an elliptical are shown, establishing the relationship between stiffness and the stress intensity factor. The effect of shear stress and torsion is not under consideration in this paper. Therefore, the stress intensity factor $K_{Q_{x}}^{I}$ and $K_{Q_{y}}^{I}$ is approximately equal to the value under pure bending. 


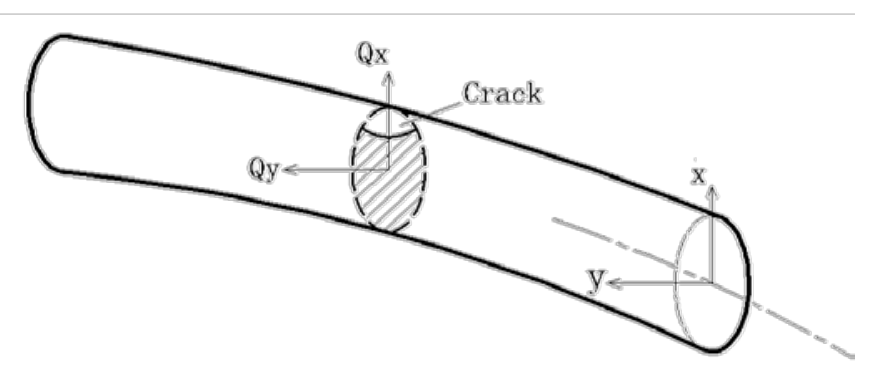

Fig.1 Forces acting on the shaft with a crack

Fig.2 shows the elliptical crack cross-section. $\mathrm{D}$ are the diameter of the shaft. The characteristic parameters are the following: crack length $\alpha=a / D$, crack shape factor $\beta=a / b$ and the relative position along the crack $\gamma=w / h h$.

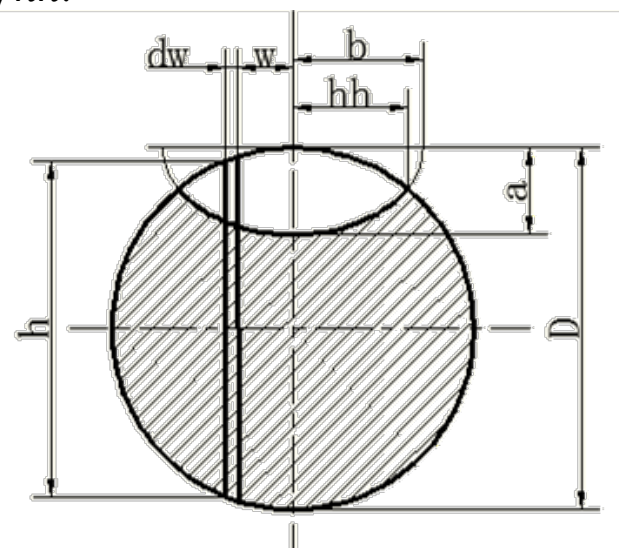

Fig.2 Elliptical crack cross-section

The stress intensity factor $K_{Q_{x}}^{I}$ due to $Q_{x}$ is approximately equal to the value for the under bending Mode I,

$$
K_{Q_{x}}^{I}=\sigma_{x} \sqrt{\pi a} K_{I, x}(\alpha, \beta, \gamma)
$$

Where

$$
\begin{aligned}
& \sigma_{x}=\frac{Q_{x} L}{4} \frac{h}{2} / \frac{\pi D^{4}}{64} \\
& K_{I, x}(\alpha, \beta, \gamma)=\sum_{i=0}^{2} \sum_{j=0}^{6} \sum_{k=0}^{2} N_{i, j, k} \beta^{i} \alpha^{j} \gamma^{k}
\end{aligned}
$$

$\mathrm{L}$ is the length of the shaft and is the depth of crack. The height of crack element $h=$ $\sqrt{D^{2}-(2 w)^{2}} \cdot \mathrm{K}_{\mathrm{I}, \mathrm{x}}(\alpha, \beta, \gamma)$ is the geometry correction factor under bending in $\mathrm{x}$ direction. A polynomial expression and its parameters can be found in Shin and Cai [8].Among that, $\alpha, \beta, \gamma$ mean the dimensionless crack depth, shape factor and relative position.

For the transverse force $Q_{y}$, the stress intensity $K_{Q_{y}}^{I}$ :

$K_{Q y}^{I}=\sigma_{y} \sqrt{\pi a} K_{I, y}(\alpha, \beta, \gamma)$

Where

$\sigma_{y}=\frac{Q_{y} L}{4} w / \frac{\pi D^{4}}{64}$

$K_{I, x}(\alpha, \beta, \gamma)=\sum_{i=0}^{2} \sum_{j=0}^{6} \sum_{k=0}^{2} Q_{i, j, k} \beta^{i} \alpha^{j} \gamma^{k}$

$\mathrm{K}_{\mathrm{I}, \mathrm{x}}(\alpha, \beta, \gamma)$ Is the geometry correction factor under bending in y direction, which can get by using a multi-parameter fitting expression in Ref. [9]? And $\alpha, \beta, \gamma$ mean the dimensionless crack depth, shape factor and relative position.

The total stress intensity factor $K^{I}$ :

$K^{I}=K_{Q_{x}}^{I}+K_{Q_{x}}^{I}$

The additional deflections due to the crack show as

$\Delta_{\text {crack }}^{x}=\frac{2}{E} \int_{0}^{a} K^{I} \frac{\partial K_{Q_{x}}^{I}}{\partial Q_{x}} d A \quad \Delta_{\text {crack }}^{y}=\frac{2}{E} \int_{0}^{a} K^{I} \frac{\partial K_{Q_{y}}^{I}}{\partial Q_{y}} d A$

For a completely open crack, the area integrations perform as: 
$\int_{0}^{A}() d A=\int_{-\sqrt{a(D-a)}}^{\sqrt{a(D-a)}} \int_{0}^{a}() d a d w$

And the total deflection can be expressed as follows:

$\Delta^{x}=Q_{x} g_{1}+Q_{y} g_{2}, \quad \Delta^{y}=Q_{x} g_{3}+Q_{y} g_{4}$

Where flexibility $g_{1}, g_{2}, g_{3}$, and $g_{4}$ are:

$g_{1}=\frac{L^{3}}{48 E I}+\iint \frac{128 L^{2} h^{2} a}{E \pi D^{8}} K_{I, x}^{2}(\alpha, \beta, \gamma) d a d w$

$g_{2}=g_{3}=\iint \frac{256 L^{2} h w a}{E \pi D^{8}} K_{I, x}(\alpha, \beta, \gamma) K_{I, y}(\alpha, \beta, \gamma) d a d w$

$g_{4}=\frac{L^{3}}{48 E I}+\iint \frac{512 L^{2} w^{2} a}{E \pi D^{8}} K_{I, y}{ }^{2}(\alpha, \beta, \gamma) d a d w$

$\mathrm{E}$ and I represent the modulus of elasticity and moment of inertia. $K_{I, x}(\alpha, \beta, \gamma)$ and $K_{I, y}(\alpha, \beta, \gamma)$ Are mentioned in equation (3) and (6). Assuming $\Delta^{x}$ and $\Delta^{y}$ as $\mathrm{x}$ and y respectively,

$Q_{x}=\left(g_{4} x-g_{2} y\right) /\left(g_{1} g_{4}-g_{2}^{2}\right), \quad Q_{y}=\left(-g_{2} x-g_{1} y\right) /\left(g_{1} g_{4}-g_{2}^{2}\right)$

On the other hand,

$Q_{x}=k_{x} x+k_{x y} y, \quad Q_{y}=k_{y} y+k_{y x} x$

Solving for $Q_{x}$ and $Q_{y}$ in equation (14) and (15), one can have:

$k_{x}=\frac{g_{4}}{g_{1} g_{4}-g_{2}^{2}} \quad k_{x y}=k_{y x}=\frac{-g_{2}}{g_{1} g_{4}-g_{2}^{2}}, \quad k_{y}=\frac{g_{1}}{g_{1} g_{4}-g_{2}^{2}}$

\section{Crack opening and closing behavior}

From the equations previously, the evaluations of $\mathrm{k}_{\mathrm{x}}, \mathrm{k}_{\mathrm{xy}}$ and $\mathrm{k}_{\mathrm{y}}$ require the value of $\mathrm{K}^{\mathrm{I}}$ at each point along the crack front. When the shaft rotates with a constant speed, the elliptical crack would be opened at its lower position, closed at upper position and partially opened at the rest due to gravitational force, called "breathing effect". The total intensity factor $\mathrm{K}^{\mathrm{I}}$ at each point determines whether the crack is open or crack.

The calculation flow of stiffness is firstly judge whether the $\mathrm{K}^{\mathrm{I}}$ is beyond zero to define the integration limits Crack Closure Line Position (CCLP), and then calculate the flexibility and stiffness at each degree when rotating.

\section{Computation and discussions}

Fig.3 and Fig.4 shows the time-varying stiffness in one full revolution. The geometrical and material properties of the shaft are: massm $=0.6 \mathrm{~kg}$, total length $\mathrm{L}=700 \mathrm{~mm}$, diameterD $=15 \mathrm{~mm}$, and Young's modulus $E=2.1 * 10^{11} \mathrm{~Pa}$, evidently, when the crack depth factor $\alpha=0.3$, increasing the value of $\beta$ would cause extra stiffness reduce. When the crack shape factor $\beta=0.5$, increasing $\alpha$ also makes the stiffness values reduce. The whole revolution can be separated to three parts. One is full stiffness, one is the stiffness of full open region, and the rest is changing parts.

It seems to be reasonable to assume that the stiffness have the identified relationship with elliptical crack depth and factor. A switching crack model is introduced to clarify the opening and closing phenomenon of an elliptical crack. Stress intensity factor within parameters fitting geometry correction factor produce a better approximation results. Simulation results suggest that the method is effective to calculate stiffness. And based on this, dynamic instability behavior and steady-state response could be studied accurately. 


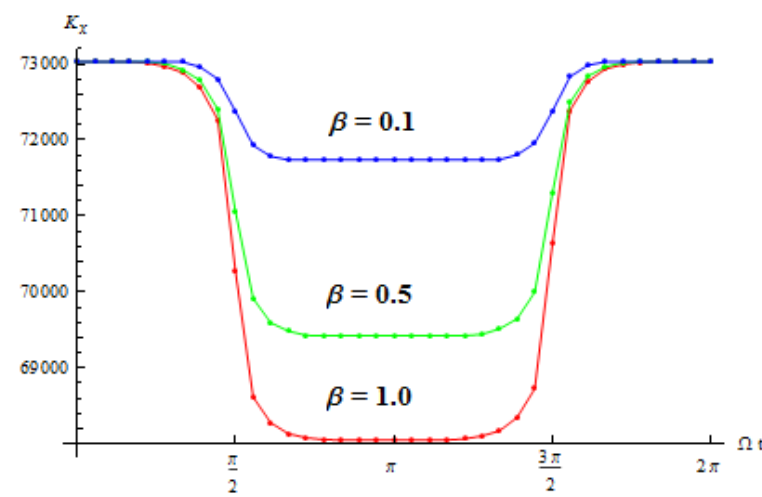

Fig.3 variation of stiffness for different $\beta$

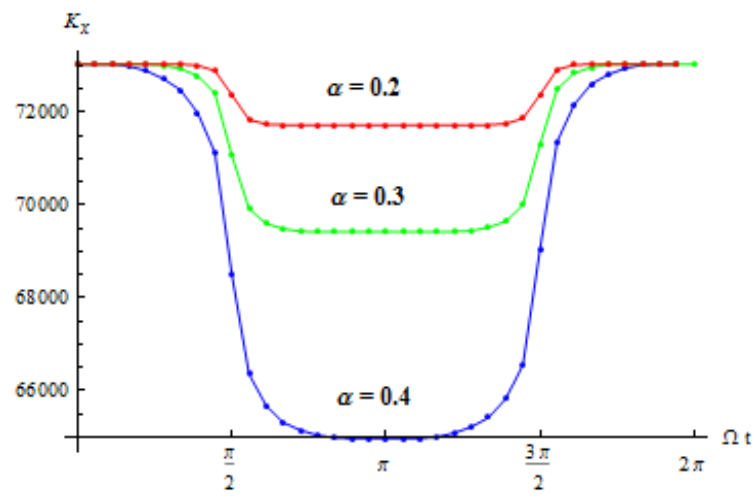

Fig.4 variation of stiffness for different $\alpha$

\section{References}

[1] A.D. Dimarogonas, S.A. Paipetis, Analytical Methods in Rotor Dynamics, Applied Science Publishers, London, New York, 1983.

[2] C.A. Papadopoulos, A.D. Dimarogonas, Coupled longitudinal and bending vibrations of a rotating shaft with an open crack, Journal of Sound and Vibration 117 (1) (1987) 81-93.

[3] J. Toribio, N. Alvarez, B. Gonzalez, J.C. Matos, A critical review of stress intensity factor solutions for surface cracks in round bars subjected to tension loading, Engineering Failure Analysis 16 (3) (2009) 794-809.

[4] O S JUN, H J EUN. Modelling and vibration analysis of a simple rotor with a breathing crack [J]. Journal of Sound and Vibration, 155(2) (1992) 273-290.

[5] L.Rubio, B Munoz-Abella, Flexibility coefficients of a shaft with an elliptical front crack. In: Institution of Mechanical Engineers -9th International Conference on Vibrations in Rotating Machinery, Exeter, UK, 2008, p. 657-667.

[6] L.Rubio, B Munoz-Abella, G. Loaiza, Static behavior of a shaft with an elliptical crack. Mech. Syst. Signal Process.25 (2011) 674-1686.

[7] C.A. Papadopoulos, The strain energy release approach for modeling cracks in rotors: a state of the art review. Mechanical Systems and Signal Processing, 22 (2008) 763-789.

[8] C.S. Shin, C.Q. Cai, Experimental and finite element analyses on stress intensity factors of an elliptical surface crack in a circular shaft under tension and bending. Int. J. Fract. 129 (2004) 239264.

[9] A. Carpinteri, R. Brighenti, A. Spagnoli, Surface flaws in cylindrical shafts under rotary bending. Fatigue Fract. Eng. Mater. Struct. 21 (1998) 1027-1035. 